\title{
Reprodutibilidade e validade de um questionário de frequência alimentar por grupos de alimentos, em adultos da Região Metropolitana de Porto Alegre, Brasil ${ }^{1}$
}

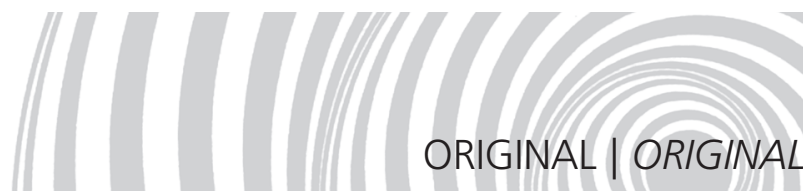

Reproducibility and validity of a food frequency

questionnaire based on food groups, in adult

population of the metropolitan region of

Porto Alegre, Brazil

Fátima Carina de Souza MACHADO²

Ruth Liane HENN²

Maria Teresa Anselmo OLINTO²

Luiz Antonio dos ANJOS 3

Vivian WAHRLICH ${ }^{4}$

William WAISSMANN ${ }^{5}$

RE S U M O

\section{Objetivo}

Testar reprodutibilidade e validade por grupos de alimentos de um questionário de frequência alimentar, desenvolvido para indivíduos adultos residentes no Sul do Brasil.

\section{Métodos}

Dois questionários de frequência alimentar e três inquéritos recordatórios de 24 horas foram aplicados a 128 participantes, com idade entre 20 e 69 anos. Os alimentos foram agrupados em 19 grupos previamente definidos. As análises foram realizadas com dados brutos e corrigidos pela energia.

\footnotetext{
1 Artigo elaborado a partir da dissertação de FCS MACHADO, intítulada "Reprodutibilidade e validade de um questionário de frequência alimentar baseado em grupos de alimentos, em população adulta da região metropolitana de Porto Alegre, RS". Universidade do Vale do Rio dos Sinos; 2010. Apoio: Coordenação de Aperfeiçoamento de Pessoal de Nível Superior, Edital PROCAD 01/2005, nº 0257052

2 Universidade do Vale do Rio dos Sinos, Programa de Pós-Graduação em Saúde Coletiva. Av. Unisinos, 950, Bairro Cristo Rei, 93022-000, São Leopoldo, RS, Brasil. Correspondência para/Correspondence to: RL HENN. E-mail: <ruthenn@unisinos.br>.

3 Universidade Federal Fluminense, Programa de Pós-Graduação em Ciências Médicas. Niterói, RJ, Brasil.

${ }^{4}$ Universidade Federal Fluminense, Faculdade de Nutrição. Niterói, RJ, Brasil.

5 Fundação Oswaldo Cruz, Escola Nacional de Saúde Pública Sergio Arouca. Rio de Janeiro, RJ, Brasil.
} 
66 FCS MACHADO et al.

\begin{abstract}
Resultados
Apenas nas estimativas de ingestão dos grupos de folhosos e de leguminosas observaram-se diferenças estatisticamente significantes entre os dois questionários de frequência alimentar. Os coeficientes de correlação, corrigidos pela energia, entre os questionários variaram de 0,53 (embutidos e presuntos) a 0,85 (bebidas alcoólicas e leite e derivados light). Quanto à validade, ambos os questionários produziram tanto estimativas de ingestão mais altas como mais baixas do que a média dos inquéritos recordatórios de 24 horas. O coeficiente de correlação médio entre cada questionário e os inquéritos recordatórios de 24 horas foi de 0,39 para o questionário de frequência alimentar 1, e de 0,40 para o questionário de frequência alimentar 2. Os coeficientes de correlação foram iguais ou maiores que 0,40 para 9 grupos alimentares no questionário de frequência alimentar 1, e para 11 grupos no questionário de frequência alimentar 2. Os percentuais médios de classificação no mesmo terço, pelos dois métodos, foram $49 \%$ para o questionário de frequência alimentar 1, e $48 \%$ para o questionário de frequência alimentar 2. Os valores de Kappa ponderado variaram de -0,04 (bebidas não-alcoólicas) a 0,69 (leite e derivados light), considerando ambos os questionários.
\end{abstract}

\title{
Conclusão
}

O questionário de frequência alimentar baseado em grupos de alimentos representa uma boa alternativa para avaliar o hábito alimentar, com a vantagem de ter uma lista reduzida de itens alimentares.

Termos de indexação: Adulto. Estudos de validação. Inquéritos sobre dietas. Reprodutibilidade dos testes.

\section{A B S T R A C T}

\section{Objective}

To test reproducibility and validity of a food frequency questionnaire based in food groups developed for adults living in Southern Brazil.

\section{Methods}

Two food frequency questionnaire (food frequency questionnaire 1 and food frequency questionnaire 2) and three 24-hour dietary recalls (24h-R) were applied to 128 participants (20-69 years). The foods were grouped in 19 groups previously defined. Analyses were performed for crude data and adjusted for energy.

\section{Results}

Statistically significant differences in the estimates of intake between two food frequency questionnaire were observed for the group of leafy vegetables and legumes. The energy-adjusted correlation coefficients between questionnaires ranged from 0.53 (sausages and hams) to 0.85 (alcoholic beverages and low-fat dairy). As to the validity, both questionnaires showed higher and lower estimates of intake in relation to 24-hour dietary recalls. The mean correlation coefficient between each food frequency questionnaire and $24 h-R$ was 0.39 (food frequency questionnaire 1) and 0.40 (food frequency questionnaire 2). The correlation coefficients were equal to or greater than 0.40 for nine food groups in the food frequency questionnaire 1 and for eleven food groups in the food frequency questionnaire 2. The average classification percentages in the same tertile, by the two methods, were $49 \%$ (food frequency questionnaire 1) and 48\% (food frequency questionnaire 2). The weighted kappa values ranged from -0.04 (non-alcoholic beverages) to 0.69 (milk and light derived) for both food frequency questionnaire.

\section{Conclusion}

This food frequency questionnaire based food group represents a good alternative for assessing the food habits, with the advantage of having a short list of food items.

Indexing terms: Adult. Validation studies. Diet surveys. Reproducibility of results.

\section{N T R O D U ÇÃ O}

Estima-se que, no ano de 2020, as Doenças Crônicas Não-Transmissíveis (DCNT) serão responsáveis por $75 \%$ das mortes em todo o mundo'. A dieta tem permanecido na agenda internacional de pesquisa como um dos principais determinantes dessas doenças. Por outro lado, estudos epidemiológicos de base populacional e ensaios clínicos randomizados evidenciam o papel 
da dieta na prevenção e controle de morbimortalidade².

Dentre os vários métodos existentes para avaliar a dieta, os mais frequentemente utilizados são o registro alimentar, o Inquérito Recordatório de 24 horas (IR24h) e o Questionário de Frequência Alimentar (QFA) ${ }^{3}$. A escolha de um ou outro método depende dos objetivos do estudo e da população investigada ${ }^{4}$.

No registro alimentar, todos os alimentos e bebidas ingeridos durante um ou mais dias são registrados pelo participante. Já no IR24h, o respondente é solicitado a lembrar e informar todos os alimentos e bebidas ingeridos nas 24 horas precedentes ou no dia anterior à entrevista. O QFA, por sua vez, baseia-se na frequência usual de ingestão dos alimentos contidos em uma lista, preestabelecida pelo pesquisador, por um período específico de tempo, que pode variar de um mês a vários anos. Pelas suas características, os dois primeiros métodos são mais utilizados para mensurar a ingestão atual, enquanto o QFA caracteriza a dieta usual3,4.

Como na associação entre dieta e DCNT, a exposição de interesse é a ingestão alimentar de longo prazo, o método de escolha é o QFA, sendo necessária apenas uma aplicação para se obter informações sobre a dieta habitual dos indivíduos. O registro alimentar e o IR24h, ao contrário, exigem múltiplos dias de coleta de dados para estimar a ingestão usual, o que implica maior tempo, maior carga para o respondente, maior complexidade no processamento dos dados e maior custo ${ }^{5,6}$.

Apesar das vantagens do QFA, esse instrumento necessita ter sua reprodutibilidade e validade testada na população alvo do estudo, uma vez que ele é composto de uma lista finita de alimentos ou itens alimentares, obtidas, via de regra, a partir de dados de ingestão dietética da população4.

Em geral, estudos de reprodutibilidade e validade dos QFA são baseados em energia e nutrientes $^{7-9}$. Recentemente, porém, essas ca- racterísticas têm sido avaliadas com base em grupos de alimentos ${ }^{10,11}$. No Brasil, entretanto, esses estudos ainda são escassos, especialmente com adultos $^{12}$. A abordagem, além de simplificar o QFA, pois permite reduzir a lista de alimentos, pode torná-lo mais útil na identificação de padrões alimentares, tanto protetores como de risco para DCNT, o que auxiliaria na formulação de recomendações nutricionais mais facilmente compreendidas pela população, uma vez que os indivíduos compram, preparam e ingerem alimentos, mais do que nutrientes.

O presente estudo teve como objetivo testar a reprodutibilidade e a validade por grupos de alimentos de um QFA desenvolvido para a população da Região Metropolitana de Porto Alegre (RS).

\section{M É T O D O S}

Este estudo está inserido no projeto de pesquisa "Construção e validação de um instrumento de avaliação da ingestão alimentar na população adulta" (PROCAD 01/2005, n 0257052) desenvolvido pelos Programas de Pós-Graduação da Universidade do Vale do Rio dos Sinos (UNISINOS), da Universidade Federal Fluminense (UFF) e da Escola Nacional de Saúde Pública Sérgio Arouca da Fundação Oswaldo Cruz (ENSP/FIOCRUZ).

Dados sobre a construção do questionário de frequência alimentar estão descritos nos estudos de Zanolla et al..$^{13}$ e Bonato ${ }^{14}$. Brevemente, a lista do QFA foi elaborada a partir das informações obtidas com a aplicação de IR24h em uma amostra probabilística de 1726 adultos avaliados na Pesquisa de Nutrição, Atividade Física e Saúde (PNAFS), inquérito domiciliar realizado em adultos residentes em Niterói, Rio de Janeiro, de janeiro a dezembro de 2003. Todos os alimentos citados nos IR24h por, no mínimo, 30 indivíduos, foram incluídos na lista, resultando em um QFA com 127 itens alimentares (QFA-127). Após ter sido testado, verificou-se a necessidade de ajustes e um novo QFA foi elaborado, cuja lista passou a conter 
120 itens alimentares (QFA-120), entre eles alimentos regionais.

Para o presente trabalho, foram utilizadas as informações sobre ingestão alimentar obtidas no estudo de reprodutibilidade e validade baseado em energia e nutrientes do QFA-12014. O método de referência para testar a validade foi o recordatório de 24 horas.

Os dados referem-se a uma amostra de conveniência de 128 indivíduos, de ambos os sexos, com idade entre 20 e 69 anos.

A coleta de dados foi realizada entre janeiro e abril de 2007. Foram aplicados dois QFAs (intervalo médio entre as aplicações=18 dias) e três IR24h (intervalo médio entre as aplicações $=16$ dias). Cada participante foi entrevistado quatro vezes: $1^{\mathrm{a}}$ vez: QFA1 e IR24h1; $2^{\mathrm{a}}$ vez: questionário sobre informações demográficas e socioeconômicas; $3^{\mathrm{a}}$ vez: QFA2 e IR24h2; e $4^{\mathrm{a}}$ vez: IR24h3. Quanto ao IR24h, teve-se o cuidado para que uma das entrevistas fosse conduzida na segunda-feira, de modo a contemplar a ingestão alimentar do domingo.

As entrevistas foram realizadas por entrevistadores treinados. As codificações dos questionários foram feitas pelos próprios entrevistadores e revisadas pelos supervisores. Para garantir a qualidade da coleta dos dados, os supervisores permaneceram nos locais da coleta a maior parte do tempo e, periodicamente, realizavam reuniões para esclarecimento de dúvidas.

Os dados demográficos e socioeconômicos foram digitados no Epi Info versão 6.0 (Centers for Disease Control and Prevention, Atlanta, Estados Unidos), com dupla entrada e validação. Os QFA e os IR24h foram digitados em planilhas de Excel, em templates desenvolvidos para esse propósito. Todos os dados de ingestão alimentar foram transformados em gramas/dia.

Os grupos de alimentos foram constituídos com base no QFA-120 e na lista reduzida de alimentos obtida por Anjos et al. ${ }^{15}$. Nesse estudo, os autores identificaram, a partir dos alimentos referidos na PNAFS, os itens alimentares que mais contribuíram para a ingestão de energia e nutrien- tes (65 itens). Também se considerou a semelhança nutricional entre os alimentos e as características que distinguem certos alimentos como, por exemplo, cereais e cereais integrais. No final, chegou-se a 19 grupos: bebidas alcoólicas; bebidas não-alcoólicas; biscoitos e bolos; carnes brancas; carnes vermelhas; cereais; cereais integrais; doces e guloseimas; embutidos e presunto; folhosos; frutas e suco natural; gorduras; leguminosas; leite e derivados integrais; leite e derivados light; pães; raízes e tubérculos; salgados; sopas e legumes.

Os alimentos referidos pelos participantes nos dois QFA e nos três IR24h foram codificados de acordo com os grupos preestabelecidos. Quanto aos IR24h, se o alimento referido fosse uma preparação, como por exemplo, lasanha, a receita era desmembrada nos seus ingredientes e estes designados para os grupos alimentares correspondentes.

Os dados socioeconômicos e demográficos foram descritos como média ou proporção.

Verificou-se a normalidade dos dados de ingestão alimentar em gramas/dia pelo teste de Shapiro-Wilk. Todos os grupos alimentares apresentaram distribuição assimétrica. Várias possibilidades de transformação foram testadas sem sucesso. Dessa forma, todas as análises foram realizadas com os dados em sua escala original.

Devido à distribuição assimétrica dos dados, não foi possível fazer a correção pela energia pelo método dos resíduos, uma vez que a análise de regressão linear tem como um de seus pressupostos a normalidade da variável resposta ${ }^{16}$. Uma opção discutida por Willett ${ }^{17}$ para corrigir pela energia é a densidade de nutrientes, em que a quantidade ingerida de nutrientes é dividida pela energia total em quilocalorias ( $\mathrm{kcal}$ ). Como neste estudo os dados de ingestão referem-se a grupos alimentares, fez-se a correção pela densidade de alimentos, dividindo-se o total em gramas de cada grupo alimentar pela energia total e multiplicando-se por $1000 \mathrm{kcal}$.

Medianas de ingestão dos grupos alimentares foram calculadas para ambos os QFA e para a média dos três IR24h. As diferenças entre 
- QFA1 e o QFA2 e entre cada QFA e a média dos três IR24h foram testadas com o teste de Wilcoxon, considerando-se um nível de significância de 5\%.

Correlação de Spearman foi utilizada tanto para testar a reprodutibilidade quanto a validade relativa.

A concordância entre cada QFA e a média dos três IR24h também foi avaliada pela classificação dos indivíduos de acordo com sua distribuição em terços de ingestão dos grupos de alimentos, em ambos os métodos. Calculou-se o percentual de indivíduos classificados no mesmo terço (concordância exata) e em terços opostos (discordância). Para a análise, utilizou-se o kappa ponderado com o respectivo intervalo de confiança de 95\% (IC95\%).

As análises foram realizadas com auxílio do programa SPSS versão 18.0, para Windows (SPSS Corp., Chicago, Estados Unidos).

A pesquisa foi submetida ao Comitê de Ética da Universidade do Vale do Rio dos Sinos e aprovada pela resolução no 014/2006. Antes da aplicação do questionário, todos os procedimen- tos foram explicados, bem como foi obtida a concordância do entrevistado em participar da pesquisa, por meio da assinatura do Termo de Consentimento Livre e Esclarecido.

\section{RESULTADOS}

Ao todo, foram estudados 128 indivíduos. Segundo Willett ${ }^{4}$, uma amostra entre 100 e 200 indivíduos é suficiente para a validação de questionários de frequência alimentar, uma vez que a inclusão de mais de 200 sujeitos oferece pouca precisão adicional e que a participação inferior a 30 fornece resultados imprecisos. A amostra foi predominantemente feminina $(73 \%)$, com idade Média (M) de 38 anos (Desvio-Padrão-DP=12,8). Entre as mulheres, $44 \%$ tinham ensino superior completo ou em andamento, e 66\% viviam sem companheiro. Para os homens, esses valores foram $40 \%$ e $51 \%$, respectivamente (dados não demostrados em Tabela).

A Tabela 1 mostra as medianas e diferenças entre as medianas para os dados ajustados

Tabela 1. Medianas e diferenças entre as medianas para os valores ajustados ${ }^{1}$ de ingestão dos grupos alimentares avaliados no QFA1 e no QFA2. Região Metropolitana de Porto Alegre (RS), 2007.

\begin{tabular}{|c|c|c|c|c|}
\hline \multirow{2}{*}{ Grupo alimentar } & \multirow{2}{*}{$\begin{array}{c}\text { Mediana QFA1 (g) } \\
\left(\mathrm{P}_{25}-\mathrm{P}_{75}\right) \\
\end{array}$} & \multirow{2}{*}{$\begin{array}{c}\text { Mediana QFA2 (g) } \\
\left(\mathrm{P}_{25}-\mathrm{P}_{75}\right) \\
\end{array}$} & \multirow{2}{*}{ Diferença } & \multirow{2}{*}{$p$-valor ${ }^{2}$} \\
\hline & & & & \\
\hline Bebidas alcoólicas & $13,1 \quad(0-57,1)$ & $(0-81,6)$ & 0,6 & 0,37 \\
\hline Bebidas não-alcoólicas & $63,2(27,5-163,4)$ & $70,6(18,5-136,6)$ & $-7,4$ & 0,21 \\
\hline Biscoitos e bolos & $4,6 \quad(1,3-10,7)$ & $(1,2-9,2)$ & 0,4 & 0,57 \\
\hline Carnes brancas & $20,7 \quad(11,5-32,9)$ & $21,2 \quad(11,1-38,8)$ & $-0,5$ & 0,59 \\
\hline Carnes vermelhas & $36,5 \quad(21,5-51,0)$ & $37,3 \quad(20,5-53,9)$ & $-0,8$ & 0,46 \\
\hline Cereais & $45,9 \quad(26,9-62,0)$ & $44,3 \quad(31,1-68,3)$ & 1,6 & 0,16 \\
\hline Cereais integrais & $(0-18,0)$ & $(0-22,7)$ & 1,0 & 0,40 \\
\hline Doces e guloseimas & $16,6 \quad(9,6-31,5)$ & $17,9 \quad(9,2-30,3)$ & $-1,4$ & 0,90 \\
\hline Embutidos e presuntos & $6,9 \quad(3,2-12,4)$ & $6,2 \quad(2,1-12,7)$ & 0,7 & 0,37 \\
\hline Folhosos & $8,8 \quad(3,9-14,0)$ & $7,2 \quad(2,2-13,4)$ & 1,5 & 0,01 \\
\hline Frutas e suco natural & $102,0(46,5-178,5)$ & $89,7(45,9-199,1)$ & 12,2 & 0,84 \\
\hline Gorduras & $(0-2,3)$ & $(0-3,1)$ & $-0,1$ & 0,09 \\
\hline Leguminosas & $20,4 \quad(9,3-39,6)$ & $21,5 \quad(9,5-47,7)$ & $-1,1$ & 0,05 \\
\hline Leite e derivados integrais & $43,0 \quad(14,2-76,9)$ & $39,9 \quad(15,0-93,3)$ & 3,2 & 0,27 \\
\hline Leite e derivados light & $(0-74,9)$ & $(0-83,7)$ & 2,4 & 0,66 \\
\hline Pães & $23,7 \quad(9,6-39,7)$ & $20,9 \quad(7,6-39,7)$ & 2,8 & 0,66 \\
\hline Raízes e tubérculos & $11,5 \quad(4,8-21,0)$ & $13,0 \quad(5,2-21,8)$ & $-1,5$ & 0,29 \\
\hline Salgados & $20,0 \quad(10,0-35,6)$ & $22,4 \quad(11,6-35,8)$ & $-2,4$ & 0,69 \\
\hline Sopas e legumes & $47,7 \quad(24,1-88,8)$ & $38,6 \quad(24,2-78,0)$ & 9,1 & 0,10 \\
\hline
\end{tabular}

${ }^{1}$ Corrigido pela energia utilizando a densidade de alimento: (alimento em gramas/energia total em kcal)*1 mil kcal.

${ }^{2}$ Teste de Wilcoxon. QFA1: Questionário de Frequência Alimentar 1; QFA2: Questionário de Frequência Alimentar 2. 
obtidos nos dois QFA. Somente o grupo dos folhosos e das leguminosas apresentaram diferenças estatisticamente significantes entre os QFA.

Os coeficientes de correlação de Spearman entre os dois QFA estão descritos na Tabela 2. Todos os coeficientes foram superiores a 0,50, tanto na análise bruta quanto na ajustada. O valor mais alto foi observado no grupo das bebidas alcoólicas e no grupo do leite e derivados light, ambos com coeficiente de 0,85.

A primeira abordagem para comparar os dois métodos de avaliação da ingestão alimentar (QFA e IR24h) foi por meio dos dados de ingestão expressos pela mediana, (Tabela 3). Tanto o QFA1 quanto o QFA2 significativamente superestimaram a ingestão de 4 grupos alimentares e subestimaram a ingestão de 7 grupos em relação à média dos três IR24h. Dos grupos alimentares superestimados, três foram os mesmos em ambos os QFA (carnes vermelhas; raízes e tubérculos; salgados). Entre os grupos subestimados, seis se repetiram nos dois QFA (bebidas não alcoólicas; carnes brancas; cereais; doces e guloseimas; embutidos e presuntos; gorduras). A análise das diferenças observadas indicou que tanto o QFA1 quanto o QFA2 subestimaram a ingestão do grupo bebidas não-alcoólicas, substancialmente.

Coeficientes de correlação de Spearman para testar a validade relativa entre cada QFA e a média dos três IR24h são apresentados na Tabela 4. Os valores ajustados pela energia variaram de -0,03 (bebidas não-alcoólicas) a 0,77 (leite e derivados ligth) no QFA1, e de 0,02 (bebidas não-alcoólicas) a 0,81 (leite e derivados ligth) no QFA2. O coeficiente de correlação foi igual ou superior a 0,40 em 8 dos 19 grupos alimentares (42\%) no QFA1, e em 10 (53\%) no QFA2.

A Tabela 5 mostra a concordância entre os métodos pela classificação dos indivíduos por terços de ingestão dos grupos alimentares. O percentual médio de indivíduos classificados no mesmo terço pelos dois métodos foi de $49 \%$ no

Tabela 2. Coeficientes de correlação de Spearman entre o QFA1 e no QFA2 para dados brutos e ajustados. Região Metropolitana de Porto Alegre (RS), 2007.

\begin{tabular}{|c|c|c|c|c|}
\hline Grupo alimentar & Bruto & IC95\% & Ajustado ${ }^{1}$ & IC95\% \\
\hline Bebidas não-alcoólicas & 0,75 & $0,66-0,82$ & 0,77 & $0,69-0,83$ \\
\hline Carnes brancas & 0,59 & $0,46-0,69$ & 0,62 & $0,50-0,72$ \\
\hline Carnes vermelhas & 0,65 & $0,54-0,74$ & 0,60 & $0,49-0,70$ \\
\hline Doces e guloseimas & 0,61 & $0,49-0,71$ & 0,59 & $0,46-0,69$ \\
\hline Embutidos e presuntos & 0,54 & $0,40-0,65$ & 0,53 & $0,39-0,64$ \\
\hline Folhosos & 0,73 & $0,64-0,80$ & 0,71 & $0,61-0,79$ \\
\hline Frutas e suco natural & 0,76 & $0,67-0,82$ & 0,77 & $0,69-0,83$ \\
\hline Leite e derivados light & 0,85 & $0,79-0,89$ & 0,85 & $0,79-0,89$ \\
\hline Pães & 0,72 & $0,62-0,79$ & 0,70 & $0,60-0,78$ \\
\hline Raízes e tubérculos & 0,60 & $0,49-0,70$ & 0,60 & $0,49-0,70$ \\
\hline Salgados & 0,72 & $0,62-0,79$ & 0,70 & $0,60-0,78$ \\
\hline Sopas e legumes & 0,69 & $0,59-0,77$ & 0,72 & $0,62-0,79$ \\
\hline Média & 0,70 & $0,60-0,78$ & 0,69 & $0,59-0,77$ \\
\hline
\end{tabular}

${ }^{1}$ Corrigido pela energia utilizando a densidade de alimento: (alimento em gramas/energia total em kcal)*1 mil kcal. QFA1: Questionário de Frequência Alimentar 1; QFA2: Questionário de Frequência Alimentar 2. 
Tabela 3. Medianas e diferenças entre as medianas para os valores ajustados ${ }^{1}$ de ingestão dos grupos alimentares obtidos a partir dos QFA1 e QFA2 e da média dos três IR24h. Região Metropolitana de Porto Alegre (RS), 2007.

\begin{tabular}{|c|c|c|c|c|c|}
\hline \multirow[b]{2}{*}{ Grupo alimentar } & \multicolumn{4}{|c|}{ QFA1 } & \multirow[b]{2}{*}{$p$-valor ${ }^{2}$} \\
\hline & $\begin{array}{l}\text { QFA Mediana (g) } \\
\left(\mathrm{P}_{25}-\mathrm{P}_{75}\right)\end{array}$ & \multicolumn{2}{|c|}{$\begin{array}{l}\text { IR24h Mediana (g) } \\
\left(\mathrm{P}_{25}-\mathrm{P}_{75}\right)\end{array}$} & Diferença & \\
\hline Bebidas alcoólicas & $13,1(0-57,1)$ & 0,0 & $(0-66,6)$ & 13,1 & 0,11 \\
\hline Bebidas não-alcoólicas & $63,2(27,5-163,4)$ & $339,1($ & $(229,4-564,9)$ & $-275,9$ & $<0,01$ \\
\hline Biscoitos e bolos & $4,6 \quad(1,3-10,7)$ & 4,3 & $(0-13,6)$ & 0,3 & 0,5 \\
\hline Carnes brancas & $20,7 \quad(11,5-32,9)$ & 42,6 & $(17,6-80,1)$ & $-21,8$ & $<0,01$ \\
\hline Carnes vermelhas & $36,5 \quad(21,5-51,1)$ & 20,0 & $(3,0-42,9)$ & 16,5 & $<0,01$ \\
\hline Cereais & $45,9 \quad(26,9-62,0)$ & 58,5 & $(36,5-89,0)$ & $-12,6$ & $<0,01$ \\
\hline Cereais integrais & $(0-18,0)$ & 3,6 & $(0-25,2)$ & 2,1 & 0,64 \\
\hline Doces e guloseimas & $16,6 \quad(9,6-31,5)$ & 29,2 & $(13,0-50,6)$ & $-12,6$ & $<0,01$ \\
\hline Embutidos e presuntos & $6,9 \quad(3,2-12,4)$ & 7,5 & $(2,4-15,2)$ & $-0,5$ & 0,05 \\
\hline Folhosos & $8,8 \quad(3,9-14,0)$ & 6,4 & $(2,8-14,9)$ & 2,4 & 0,73 \\
\hline Frutas e suco natural & $102,0(46,5-178,5)$ & 96,9 & $(27,6-186,7)$ & 5,0 & 0,07 \\
\hline Gorduras & $0,5 \quad(0-2,3)$ & 3,6 & $(0,8-8,6)$ & $-3,1$ & $<0,01$ \\
\hline Leguminosas & $20,4 \quad(9,4-39,6)$ & 21,7 & $(2,5-44,0)$ & $-1,3$ & 0,31 \\
\hline Leite e derivados integrais & $43,0 \quad(14,2-76,9)$ & 59,8 & $(16,2-105,1)$ & $-16,7$ & 0,01 \\
\hline Leite e derivados light & $8,4 \quad(0-74,9)$ & 1,3 & $(0-65,5)$ & 7,1 & 0,23 \\
\hline Pães & $23,7 \quad(9,6-39,7)$ & 23,6 & $(15,2-46,6)$ & 0,1 & 0,01 \\
\hline Raízes e tubérculos & $11,5 \quad(4,8-21,0)$ & 0,0 & $(0-15,7)$ & 11,5 & $<0,01$ \\
\hline Salgados & $20,0 \quad(10,0-35,6)$ & 12,2 & $(0-42,7)$ & 7,8 & $<0,01$ \\
\hline Sopas e legumes & $47,7 \quad(24,1-88,8)$ & 39,4 & $(21,7-63,4)$ & 8,3 & 0,06 \\
\hline \multirow[b]{2}{*}{ Grupo alimentar } & \multicolumn{4}{|c|}{ QFA2 } & \multirow[b]{2}{*}{$p$-valor ${ }^{2}$} \\
\hline & $\begin{array}{c}\text { QFA Mediana (g) } \\
\left(\mathrm{P}_{25}-\mathrm{P}_{75}\right)\end{array}$ & \multicolumn{2}{|c|}{$\begin{array}{c}\text { IR24h Mediana (g) } \\
\left(\mathrm{P}_{25}-\mathrm{P}_{75}\right) \\
\end{array}$} & Diferença & \\
\hline Bebidas alcoólicas & $12,5 \quad(0-81,6)$ & 0,0 & $(0-66,6)$ & 12,5 & 0,03 \\
\hline Bebidas não-alcoólicas & $70,6(18,5-136,6)$ & 339,1 & $(229,4-564,9)$ & $-268,5$ & $<0,01$ \\
\hline Biscoitos e bolos & $4,2 \quad(1,2-9,2)$ & 4,3 & $(0-13,6)$ & $-0,1$ & 0,26 \\
\hline Carnes brancas & $21,2 \quad(11,1-38,8)$ & 42,6 & $(17,6-80,1)$ & $-21,4$ & $<0,01$ \\
\hline Carnes vermelhas & $37,3 \quad(20,5-53,9)$ & 20,0 & $(3,0-42,9)$ & 17,3 & $<0,01$ \\
\hline Cereais & $44,3 \quad(31,1-68,3)$ & 58,5 & $(36,5-89,0)$ & $-14,2$ & $<0,01$ \\
\hline Cereais integrais & $(0-22,7)$ & 3,6 & $(0-25,2)$ & 1,1 & 0,2 \\
\hline Doces e guloseimas & $17,9 \quad(9,2-30,3)$ & 29,2 & $(13,0-50,6)$ & $-11,2$ & $<0,01$ \\
\hline Embutidos e presuntos & $6,2 \quad(2,1-12,7)$ & 7,5 & $(2,4-15,2)$ & $-1,3$ & 0,02 \\
\hline Folhosos & $7,2 \quad(2,2-13,4)$ & 6,4 & $(2,8-14,9)$ & 0,8 & 0,16 \\
\hline Frutas e suco natural & $89,7(45,9-199,1)$ & 96,9 & $(27,6-186,7)$ & $-7,2$ & 0,31 \\
\hline Gorduras & $(0-3,1)$ & 3,6 & $(0,8-8,6)$ & $-3,1$ & $<0,01$ \\
\hline Leguminosas & $21,5 \quad(9,5-47,7)$ & 21,7 & $(2,5-44,0)$ & $-0,2$ & 0,07 \\
\hline Leite e derivados integrais & $39,8 \quad(15,0-93,3)$ & 59,8 & $(16,2-105,1)$ & $-19,9$ & 0,34 \\
\hline Leite e derivados light & $(0-83,7)$ & 1,3 & $(0-65,5)$ & 4,6 & 0,27 \\
\hline Pães & $20,9 \quad(7,6-39,7)$ & 23,6 & $(15,2-46,6)$ & $-2,6$ & $<0,01$ \\
\hline Raízes e tubérculos & $13,0 \quad(5,2-21,8)$ & 0,0 & $(0-15,7)$ & 13,0 & $<0,01$ \\
\hline Salgados & $22,4 \quad(11,6-35,8)$ & 12,2 & $(0-42,7)$ & 10,2 & 0,05 \\
\hline Sopas e legumes & $38,6 \quad(24,2-78,0)$ & 39,4 & $(21,7-63,4)$ & $-0,9$ & 0,42 \\
\hline
\end{tabular}

${ }^{1}$ Corrigido pela energia utilizando a densidade de alimento: (alimento em gramas/energia total em kcal)* 1 mil kcal; ${ }^{2}$ Teste de Wilcoxon. QFA1: Questionário de Frequência Alimentar 1; QFA2: Questionário de Frequência Alimentar 2; IR24h: Inquéritos Recordatórios de 24 horas.

QFA1, e 48\% no QFA2. A classificação nos terços opostos foi, em média, 13\% e 12\%, respectivamente. O valor de Kappa ponderado variou de -
0,04 a 0,65 no QFA1, e de 0,01 a 0,69 no QFA2, respectivamente, para os grupos de bebidas não-alcoólicas e de leite e derivados light. 
Tabela 4. Coeficientes de correlação de Spearman entre cada QFA1 e QFA2 e a média dos três IR24h para dados brutos e ajustados Região Metropolitana de Porto Alegre (RS), 2007.

\begin{tabular}{|c|c|c|c|c|}
\hline \multirow{2}{*}{ Grupo alimentar } & \multicolumn{3}{|c|}{ QFA1 } & \multirow[b]{2}{*}{ IC95\% } \\
\hline & Bruto & IC95\% & Ajustado ${ }^{1}$ & \\
\hline Bebidas alcoólicas & 0,59 & $0,46-0,69$ & 0,58 & $0,45-0,68$ \\
\hline Bebidas não-alcoólicas & 0,04 & $-0,13-0,21$ & $-0,03$ & $-0,20-0,14$ \\
\hline Biscoitos e bolos & 0,33 & $0,16-0,48$ & 0,33 & $0,16-0,48$ \\
\hline Carnes brancas & 0,06 & $-0,11-0,23$ & $-0,02$ & $-0,19-0,15$ \\
\hline Carnes vermelhas & 0,07 & $-0,10-0,24$ & 0,01 & $-0,16-0,18$ \\
\hline Cereais & 0,54 & $0,40-0,65$ & 0,37 & $0,21-0,51$ \\
\hline Cereais integrais & 0,73 & $0,64-0,80$ & 0,73 & $0,64-0,80$ \\
\hline Doces e guloseimas & 0,23 & $0,06-0,39$ & 0,15 & $-0,02-0,31$ \\
\hline Embutidos e presuntos & 0,38 & $0,22-0,52$ & 0,39 & $0,23-0,53$ \\
\hline Folhosos & 0,48 & $0,33-0,60$ & 0,45 & $0,30-0,58$ \\
\hline Frutas e suco natural & 0,59 & $0,46-0,69$ & 0,63 & $0,51-0,72$ \\
\hline Gorduras & 0,41 & $0,25-0,54$ & 0,39 & $0,23-0,53$ \\
\hline Leguminosas & 0,59 & $0,46-0,69$ & 0,49 & $0,34-0,61$ \\
\hline Leite e derivados integrais & 0,55 & $0,42-0,66$ & 0,55 & $0,42-0,66$ \\
\hline Leite e derivados light & 0,77 & $0,69-0,83$ & 0,77 & $0,69-0,83$ \\
\hline Pães & 0,49 & $0,34-0,61$ & 0,5 & $0,36-0,62$ \\
\hline Raízes e tubérculos & 0,26 & $0,09-0,41$ & 0,27 & $0,10-0,42$ \\
\hline Salgados & 0,39 & $0,23-0,53$ & 0,39 & $0,23-0,53$ \\
\hline Sopas e legumes & 0,44 & $0,29-0,57$ & 0,48 & $0,33-0,60$ \\
\hline Média & 0,42 & $0,27-0,54$ & 0,39 & $0,24-0,52$ \\
\hline \multirow{2}{*}{ Grupo alimentar } & \multicolumn{3}{|c|}{ QFA2 } & \\
\hline & Bruto & IC95\% & Ajustado ${ }^{1}$ & IC95\% \\
\hline Bebidas alcoólicas & 0,59 & $0,46-0,69$ & 0,58 & $0,45-0,68$ \\
\hline Bebidas não-alcoólicas & 0,12 & $-0,05-0,29$ & 0,02 & $-0,15-0,19$ \\
\hline Biscoitos e bolos & 0,34 & $0,18-0,48$ & 0,34 & $0,18-0,48$ \\
\hline Carnes brancas & 0,05 & $-0,12-0,22$ & 0,05 & $-0,12-0,22$ \\
\hline Carnes vermelhas & 0,11 & $-0,06-0,28$ & 0,08 & $-0,09-0,25$ \\
\hline Cereais & 0,51 & $0,37-0,63$ & 0,4 & $0,24-0,54$ \\
\hline Cereais integrais & 0,71 & $0,61-0,79$ & 0,72 & $0,62-0,79$ \\
\hline Doces e guloseimas & 0,27 & $0,10-0,42$ & 0,23 & $0,06-0,39$ \\
\hline Embutidos e presuntos & 0,38 & $0,22-0,52$ & 0,36 & $0,19-0,50$ \\
\hline Folhosos & 0,48 & $0,33-0,60$ & 0,49 & $0,34-0,61$ \\
\hline Frutas e suco natural & 0,62 & $0,50-0,72$ & 0,6 & $0,49-0,70$ \\
\hline Gorduras & 0,41 & $0,25-0,54$ & 0,41 & $0,25-0,54$ \\
\hline Leguminosas & 0,52 & $0,38-0,64$ & 0,47 & $0,32-0,59$ \\
\hline Leite e derivados integrais & 0,55 & $0,42-0,66$ & 0,53 & $0,39-0,64$ \\
\hline Leite e derivados light & 0,79 & $0,71-0,85$ & 0,81 & $0,74-0,86$ \\
\hline Pães & 0,55 & $0,42-0,66$ & 0,56 & $0,43-0,67$ \\
\hline Raízes e tubérculos & 0,17 & $0-0,33$ & 0,16 & $0,01-0,32$ \\
\hline Salgados & 0,28 & $0,11-0,43$ & 0,35 & $-0,19-0,49$ \\
\hline Sopas e legumes & 0,39 & $0,23-0,53$ & 0,46 & $0,31-0,59$ \\
\hline Média & 0,41 & $0,27-0,54$ & 0,4 & $0,25-0,53$ \\
\hline
\end{tabular}

${ }^{1}$ Corrigido pela energia utilizando a densidade de alimento: (alimento em gramas/energia total em kcal)* 1 mil kcal. QFA1: Questionário de Frequência Alimentar 1; QFA2: Questionário de Frequência Alimentar 2; IR24h: Inquéritos Recordatórios de 24 horas.

\section{I S C U S S Ã O}

O presente estudo testou a reprodutibilidade e validade por grupos de alimentos de um
QFA desenvolvido para adultos de ambos os sexos, residentes na Região Metropolitana de Porto Alegre (RS). A reprodutibilidade foi medida por meio da aplicação do QFA em dois momentos (teste- 
Tabela 5. Classificação dos participantes em terços de ingestão dos grupos alimentares, para valores ajustados ${ }^{1}$ pela energia, segundo QFA1 e QFA2 e a média dos três IR24h. Região Metropolitana de Porto Alegre (RS), 2007.

\begin{tabular}{|c|c|c|c|c|}
\hline \multirow{2}{*}{ Grupo alimentar } & \multicolumn{3}{|c|}{ QFA1 } & \multirow[b]{2}{*}{ IC95\% } \\
\hline & Mesmo terço (\%) & Terço oposto (\%) & Kappa & \\
\hline Bebidas alcoólicas & 58,6 & 10,9 & 0,46 & $0,34-0,58$ \\
\hline Bebidas não-alcoólicas & 31,3 & 23,4 & $-0,04$ & $-0,17-0,1$ \\
\hline Biscoitos e bolos & 43,0 & 14,1 & 0,21 & $0,07-0,35$ \\
\hline Carnes brancas & 32,8 & 24,2 & $-0,03$ & $-0,17-0,1$ \\
\hline Carnes vermelhas & 38,3 & 21,9 & 0,06 & $-0,08-0,2$ \\
\hline Cereais & 47,7 & 15,6 & 0,23 & $0,09-0,37$ \\
\hline Cereais integrais & 60,9 & 5,5 & 0,52 & $0,41-0,64$ \\
\hline Doces e guloseimas & 38,3 & 19,5 & 0,08 & $-0,06-0,22$ \\
\hline Embutidos e presuntos & 46,1 & 11,7 & 0,26 & $0,13-0,40$ \\
\hline Folhosos & 54,7 & 11,7 & 0,36 & $0,22-0,49$ \\
\hline Frutas e suco natural & 57,0 & 5,5 & 0,45 & $0,33-0,58$ \\
\hline Gorduras & 47,7 & 9,4 & 0,31 & $0,18-0,44$ \\
\hline Leguminosas & 52,3 & 8,6 & 0,36 & $0,23-0,49$ \\
\hline Leite e derivados integrais & 53,1 & 7,8 & 0,39 & $0,26-0,52$ \\
\hline Leite e derivados light & 68,8 & 1,6 & 0,65 & $0,55-0,75$ \\
\hline Pães & 52,3 & 7,0 & 0,38 & $0,26-0,51$ \\
\hline Raízes e tubérculos & 43,0 & 21,9 & 0,18 & $0,04-0,31$ \\
\hline Salgados & 47,7 & 17,2 & 0,25 & $0,11-0,39$ \\
\hline Sopas e legumes & 49,2 & 9,4 & 0,32 & $0,19-0,46$ \\
\hline Média & 48,6 & 13,0 & 0,28 & $0,20-0,37$ \\
\hline \multirow{2}{*}{ Grupo alimentar } & \multicolumn{3}{|c|}{ QFA2 } & \\
\hline & Mesmo terço (\%) & Terço oposto (\%) & Kappa & IC95\% \\
\hline Bebidas alcoólicas & 62,5 & 10,9 & 0,5 & $0,37-0,62$ \\
\hline Bebidas não-alcoólicas & 35,9 & 23,4 & 0,01 & $-0,13-0,15$ \\
\hline Biscoitos e bolos & 45,3 & 15,6 & 0,22 & $0,08-0,36$ \\
\hline Carnes brancas & 35,9 & 22,7 & 0,03 & $-0,11-0,16$ \\
\hline Carnes vermelhas & 39,8 & 19,5 & 0,11 & $-0,03-0,25$ \\
\hline Cereais & 45,3 & 10,2 & 0,27 & $0,13-0,40$ \\
\hline Cereais integrais & 60,9 & 3,9 & 0,54 & $0,43-0,65$ \\
\hline Doces e guloseimas & 42,2 & 15,6 & 0,17 & $0,03-0,31$ \\
\hline Embutidos e presuntos & 43,8 & 11,7 & 0,23 & $0,10-0,37$ \\
\hline Folhosos & 52,3 & 8,6 & 0,37 & $0,24-0,50$ \\
\hline Frutas e suco natural & 45,3 & 3,1 & 0,35 & $0,23-0,47$ \\
\hline Gorduras & 49,2 & 10,2 & 0,32 & $0,19-0,45$ \\
\hline Leguminosas & 49,2 & 10,2 & 0,31 & $0,18-0,44$ \\
\hline Leite e derivados integrais & 53,1 & 10,2 & 0,36 & $0,23-0,49$ \\
\hline Leite e derivados light & 73,4 & 2,3 & 0,69 & $0,60-0,79$ \\
\hline Pães & 51,6 & 4,7 & 0,4 & $0,28-0,52$ \\
\hline Raízes e tubérculos & 35,2 & 23,4 & 0,08 & $-0,05-0,21$ \\
\hline Salgados & 49,2 & 15,6 & 0,29 & $0,15-0,42$ \\
\hline Sopas e legumes & 48,4 & 7,8 & 0,33 & $0,20-0,46$ \\
\hline Média & 48,3 & 12,1 & 0,29 & $0,21-0,38$ \\
\hline
\end{tabular}

${ }^{1}$ Corrigido pela energia utilizando a densidade de alimento: (alimento em gramas/energia total em kcal)*1 mil kcal. QFA1: Questionário de Frequência Alimentar 1; QFA2: Questionário de Frequência Alimentar 2; IR24h: Inquéritos Recordatórios de 24 horas.

-reteste), enquanto a validade foi avaliada comparando-se o QFA com a média de três IR24h.

Na comparação das medianas de ingestão, a diferença entre os QFA foi observada apenas para dois grupos (folhosos e leguminosas). Nagel et al. ${ }^{18}$ avaliaram a reprodutibilidade, a longo prazo, de um QFA, entre os participantes do EPICHeidelberg (European Prospective Investigation 
into Cancer and Nutrition-Heidelberg), aplicando um questionário na linha de base e outro no quinto e oitavo anos de seguimento. Em apenas 6, dos 17 grupos avaliados, os autores observaram diferenças entre as duas aplicações do QFA. Já no estudo de reprodutibilidade conduzido com mulheres chinesas, diferenças significativas entre as duas aplicações do QFA foram observadas para a maioria dos grupos analisados $(78 \%)^{19}$. Em estudo realizado com 35 indivíduos, em São Paulo, Brasil, observou-se que as diferenças entre as duas aplicações do QFA foram para 20\%, apenas, dos grupos analisados; nesse estudo, entretanto, não houve ajuste pela energia ${ }^{12}$.

Quando a reprodutibilidade foi analisada por meio dos coeficientes de correlação de Spearman, todos os grupos alimentares, tanto brutos quanto corrigidos pela energia, apresentaram coeficientes acima de 0,50 , resultados semelhantes aos encontrados por Parr et al. ${ }^{20}$, para dados brutos. Em geral, os dados encontrados na literatura mostram coeficientes inferiores aos deste estudo ${ }^{11,18,21}$. Coeficientes de correlação entre 0,40 e 0,70 são considerados indicativos de boa reprodutibilidade do QFA ${ }^{22}$.

Sabe-se contudo que, na avaliação da reprodutibilidade do QFA, o intervalo de tempo entre as aplicações deve ser considerado. Intervalos muito curtos, de poucos dias, levam os participantes a repetirem as respostas dadas no primeiro QFA, produzindo correlações altas, porém espúrias ${ }^{23,24}$. Por outro lado, intervalos longos podem gerar variações nas respostas devido a mudanças reais na ingestão alimentar, levando a coeficientes mais baixos ${ }^{4}$, como encontrado em alguns estudos ${ }^{23,24}$. Neste estudo, o intervalo médio entre as duas aplicações do QFA foi de 18 dias, de acordo com o que recomenda a literatura, entre 15 e 45 dias $^{25}$.

No presente estudo, a validade do QFA foi testada com diferentes abordagens, conforme recomendado por Cade et al. ${ }^{25}$. A primeira delas foi a comparação das medianas de ingestão dos dois métodos (QFA e IR24h). Ambos os QFA significativamente superestimaram a ingestão de 4 grupos alimentares e subestimaram a ingestão de 7 grupos, não havendo diferenças entre os 8 grupos restantes. Dados da literatura mostraram que - QFA tanto superestimou quanto subestimou a ingestão em relação ao método de referência e, embora os grupos alimentares super e subestimados não fossem necessariamente os mesmos nos diferentes estudos, observou-se uma tendência de superestimação para o grupo das frutas 12,21,26, resultado não encontrado neste trabalho. Já a grande subestimação na ingestão de bebidas não-alcoólicas, observada tanto no QFA1 quanto no QFA2, foi consistente com os resultados da literatura ${ }^{10,27}$.

A validade do QFA, avaliada por meio da correlação de Spearman, mostrou coeficientes superiores a 0,40 para a maioria dos grupos alimentares não ajustados, em ambos os QFA. Alguns coeficientes, porém, diminuíram após a correção pela energia, semelhante aos achados de outros estudos de validação ${ }^{10,21}$. A correção pela energia é feita com base no pressuposto de que cada entrevistado relata a ingestão de maneira similar nos dois métodos ${ }^{28}$. Se isso não ocorre, o ajuste pode diminuir os coeficientes ${ }^{12}$. É importante salientar, entretanto, que neste estudo não foi possível utilizar o método dos resíduos para fazer a correção pela energia, como nos demais trabalhos, devido à natureza dos dados. Assim, optou-se pela abordagem da densidade de alimentos como uma proxy da densidade de nutrientes, esta última uma alternativa que tem muitas vantagens, dentre elas a possibilidade de ser calculada diretamente sem o uso de qualquer modelo estatístico, além de ser familiar aos nutricionistas e poder ser utilizada para a formulação de orientações nutricionais ${ }^{17}$. A utilização desse método em análises epidemiológicas, no entanto, pode ser limitada quando a ingestão de energia total está associada com alguma doença. Nessa situação, os nutrientes ingeridos podem ser confundidos pela energia total ingerida; assim, a divisão por esta última levaria o confundimento para o sentido oposto, um resultado indesejável para essa razão ${ }^{17}$. Esses aspectos, possivelmente, 
também se aplicam à abordagem da densidade de alimentos.

Em estudos epidemiológicos, a categorização da ingestão de alimentos/grupo de alimentos permite o cálculo de medidas de associação. Dessa forma, a habilidade de um questionário em classificar corretamente os indivíduos segundo o seu nível de ingestão contribui para a obtenção de estimativas de risco acuradas ${ }^{29}$. Neste estudo, o percentual de indivíduos classificados no mesmo terço de ingestão variou de $31,3 \%$ a $68,8 \%$ no QFA1, e de $35,2 \%$ a $73,4 \%$, no QFA2, resultados semelhantes ao de Matarazzo et al. ${ }^{12}$ (31,0\% a $74,0 \%)$. Contudo, somente quatro grupos alimentares no QFA1 (bebidas alcoólicas; cereais integrais; frutas e suco natural; leites e derivados light) e quatro no QFA2 (bebidas alcoólicas; cereais integrais; leites e derivados light; pães) apresentaram valores de Kappa iguais ou superiores a 0,40, o que corresponde a uma concordância de moderada a alta. No estudo de Voci et al. ${ }^{30}$, realizado com adolescentes, os grupos alimentares com esses valores foram: leite e derivados; biscoitos recheados; verduras e legumes; e feijão. Nesta análise, entretanto, é importante levar em consideração que a classificação dos entrevistados de acordo com a distribuição de ingestão nos dois métodos tanto pode agrupar indivíduos com níveis de ingestão diferentes na mesma categoria, como colocar em categorias distintas aqueles com níveis de ingestão similares, caso estejam muito próximos do ponto de corte, especialmente se a amostra é pequena ${ }^{31}$, como foi o caso deste estudo.

Apesar da validade satisfatória, algumas limitações devem ser consideradas. No presente estudo não foi possível corrigir pela variabilidade intraindividual no método de referência, uma vez que essa correção é feita com base nas variâncias intra e inter-indivíduos obtidas na análise de variância, a qual exige dados normalmente distribuídos. Sabe-se que essa variabilidade, quando não corrigida, pode atenuar os coeficientes de correlação ${ }^{32}$. No entanto, mesmo não corrigidos, os coeficientes obtidos foram, em geral, mais altos do que aqueles vistos nos estudos de validação por nutrientes ${ }^{7}, 14$.
Outra limitação é o método de referência escolhido. Como tanto o IR24h quanto o QFA dependem da memória do entrevistado, as fontes de erro em ambos os métodos podem ser correlacionadas ${ }^{32}$. No entanto, como o período de referência para cada método é bem distinto (24 horas vs 1 mês), acredita-se que esse tipo de erro esteja minimizado. Optou-se por não se utilizar o RA, uma vez que ele demanda maior esforço dos participantes e pode provocar alterações na ingestão alimentar para facilitar o registro ${ }^{32}$. Além disto, os estudos que utilizaram o RA como método de referência mostraram resultados bastante semelhantes aos encontrados por aqueles que utilizaram o IR24h. Erkkola et al. ${ }^{27}$ utilizaram 10 registros alimentares, encontrando correlações de 0,04 a 0,86 para validade. Já no estudo realizado por Matarazzo et al. ${ }^{12}$, utilizando apenas um único IR24h, a validade variou de 0,05 a 0,71. Dessa forma, o IR24h tem sido amplamente utilizado por ser de mais fácil aplicação, demandar menor esforço do participante e não exigir habilidades especiais do entrevistado 3 . O número de IR24h utilizados pode não ter sido suficiente para descrever a ingestão usual média dos participantes, especialmente com relação àqueles alimentos não ingeridos numa base diária. Entretanto, maior número de replicatas exigiria mais esforço dos entrevistados, resultando em abandono do estudo ${ }^{14}$.

Como no estudo de validação por nutrientes, não se pode descartar o viés do voluntariado, uma vez que se utilizou amostra de conveniência. Isso poderia contribuir para a ocorrência de correlações mais fortes do que na população em geral, uma vez que existe uma motivação diferenciada nos voluntários para participar de pesquisas.

Outra limitação diz respeito à escolaridade da amostra que, neste estudo, foi elevada. Assim, desconhece-se qual seria o desempenho deste QFA em população com baixa escolaridade.

\section{O N CLUSÃO}

Os achados deste estudo indicam boa reprodutibilidade para todos os grupos alimentares 
e validade satisfatória para a maioria dos grupos analisados, quando são considerados os métodos mais usualmente empregados na literatura, tais como os coeficientes de correlação de Spearman e a classificação dos participantes em terços de ingestão. A utilização de um QFA baseado em grupos de alimentos pode ser uma boa alternativa para avaliar o hábito alimentar, com a vantagem de ter uma lista reduzida de itens alimentares. Além disso, pode ser mais interessante do ponto de vista da informação para a população, pois as recomendações nutricionais baseadas em alimentos ou grupo de alimentos são mais facilmente compreendidas, favorecendo a adesão a elas. Entretanto, como a ingestão alimentar não é estimada sem erro, mais estudos devem ser realizados, com o intuito de desenvolver metodologias que minimizem possíveis distorções nessas estimativas.

\section{COLABORADORES}

Todos os autores participaram de todas as fases da pesquisa do artigo.

\section{REFERÊ NCIAS}

1. World Health Organization. Diet, nutrition and the prevention of chronic diseases. Report of a Joint FAO/WHO/UNU Expert Consultation. Geneva: WHO; 2003. WHO Technical Report Series, 916.

2. World Health Organization. Reducing risks, promoting healthy life. Geneva: WHO; 2002. World Health Report.

3. Thompson FE, Byers T. Dietary assessment resource manual. J Nutr. 1994; 124(11):2245-70.

4. Willett WC. Invited commentary: comparison of food frequency questionnaires. Am J Epidemiol. 1998; 148(12):1157-9.

5. Lopes ACS, Caiaffa WT, Mingoti SA, Lima-Costa MFF. Ingestão alimentar em estudos epidemiológicos. Rev Bras Epidemiol. 2003; 6(3):209-19.

6. Cavalcanti AAM, Priore SE, Franceschini SCC. Estudos de consumo alimentar: aspectos metodológicos gerais e o seu emprego na avaliação de crianças e adolescentes. Rev Bras Saúde Mater Infant. 2004; 4(3):229-40.
7. Henn RL, Moreira LB, Fuchs FD, Fuchs SC. Development and validation of a food frequency questionnaire (FFQ-Porto Alegre) for the adolescent, adult and elderly population from southern Brazil. Cad Saúde Pública. 2010; 26(11): 2068-79.

8. Lima FEL, Slater B, Latorre MRO, Fisberg RM. Validade de um questionário quantitativo de freqüência alimentar desenvolvido para população feminina no Nordeste do Brasil. Rev Bras Epidemiol. 2007; 10(4):483-90.

9. Birgisdottir BE, Kiely M, Martinez JA, Thorsdottir I. Validity of a food frequency questionnaire to assess intake of seafood in adults in three European countries. Food Control. 2008; 19(7):648-53.

10. Sasaki S, Kobayaski M, Tsugane S. Validity of a selfadministered food frequency questionnaire used in the 5-year follow-up survey of the JPHC Study Cohort I: comparision with dietary records for food groups. J Epidemiol. 2003; 13(1):51-6.

11. Sasaki S, Ishihara J, Tsugane S. Reproducibility of a self-administered food frequency questionnaire used in the 5-year follow-up survey of the JPHC study cohort I to assess food and nutrient intake. J Epidemiol. 2003; 13(1):115-24.

12. Matarazzo HCZ, Marchioni DML, Figueiredo RAO, Slater B, Neto JE, Filho VW. Reprodutibilidade e validade do questionário de freqüência de consumo alimentar utilizado em estudo caso-controle de câncer oral. Rev Bras Epidemiol. 2006; 9(3):316-24.

13. Zanolla AF, Olinto MTA, Henn RL, Wahrlich V, Anjos LA. Avaliação de reprodutibilidade e validade de um questionário de freqüência alimentar em adultos residentes em Porto Alegre, Rio Grande do Sul, Brasil. Cad Saúde Pública. 2009; 25(4):840-8.

14. Bonatto S. Reprodutibildade e validade de um questionário de freqüência alimentar para população adulta da região metropolitana de Porto Alegre/RS [mestrado]. São Leopoldo: Universidade do Vale do Rio dos Sinos; 2009.

15. Anjos LA, Wahrlich V, Vasconcellos MTL, Souza DR, Olinto MTA, Waissmann W, et al. Desenvolvimento de questionário de freqüência alimentar em amostra probabilística de adultos de Niterói, Rio de Janeiro. Cad Saúde Pública. 2010; 26(11):2196-204.

16. Altman DG. Practical statistics for medical researches. London: Chapman \& Hall; 1997.

17. Willett WC, Howe GR, Kushi LH. Adjustment for total energy intake in epidemiologic studies. Am J Clin Nutr. 1997; 65(4):1120-8.

18. Nagel G, Zoller D, Ruf T, Rohrmann S, Linseisen J. Long-term reproducibility of a food-frequency questionnaire and dietary changes in the European 
Prospective Investigation into Cancer and Nutrition (EPIC)-Heidelberg cohort. Br J Nutr. 2007; 98(1): 194-200.

19. Shu XO, Yang G, Liu D, Kushi L, Wen W, Gao YT, et al. Validity and reproducibility of the food frequency questionnaire used in the Shanghai Women's Health Study. Eur J Clin Nutr. 2004; 58(1): 17-23.

20. Parr CL, Veierod MB, Laake P, Lund E, Hjartaker A. Test-retest reproducibility of a food frequency questionnaire (FFQ) and estimated effects on disease risk in the Norwegian Women and Cancer Study (NOWAC). Nutr J. 2006; 5(4):361-70.

21. Trinidad Rodríguez I, Fernández Ballart J, Cucó Pastor G, Biarnés Jordà E, Arija Val V. Validación de un cuestionario de frecuencia de consumo alimentario corto: reproducibilidad y validez. Nutr Hosp. 2008; 23(3):242-52.

22. Willett WC. Future directions in the development of food-frequency questionnaires. Am J Clin Nutr. 1994; 59(1):171-4.

23. Salvo VLMA, Gimeno SGA. Reprodutibilidade e validade do questionário de freqüência de consumo de alimentos. Rev Saúde Pública. 2002; 36(4): 505-12.

24. Roddam AW, Spencer E, Banks E, Beral V, Reeves G, Appleby P, et al. Reproducibility of a short semiquantitative food group questionnaire and its performance in estimating nutrient intake compared with a 7-day diet diary in the Million Women Study. Public Health Nutr. 2005; 8(2):201-13.

25. Cade J, Thompson R, Burley V, Warm D. Development, validation and utilisation of food- frequency questionnaires: a review. Public Health Nutr. 2002; 5(4):567-87.

26. Marks GC, Hughes MC, Pols JCVD. Relative validity of food intake estimates using a food frequency questionnaire is associated with sex, age, and other personal characteristics. J Nutr. 2006; 136(2):459-65.

27. Erkkola M, Karppinen M, Javanainen J, Räsänen L, Knip M, Virtanen SM. Validity and reproducibility of a food frequency questionnaire for pregnant finnish women. Am J Epidemiol. 2001; 154(5): 466-76.

28. Flegal KM, Larkin FA. Partitioning macronutrient intake estimates from a food frequency questionnaire. Am J Epidemiol. 1990; 131(6):1046-58.

29. Sevak L, Mangtani P, McCormack V, Bhakta D, Kassam-Khamis T, Silva IS. Validation of a food frequency questionnarire to assess macro- and micro-nutrient intake among South Asians in the United Kingdom. Eur J Nutr. 2004; 43(3):160-8.

30. Voci SM, Enes CC, Slater B. Validação do Questionário de Freqüência Alimentar para Adolescentes (QFAA) por grupos de alimentos em uma população de escolares. Rev Bras Epidemiol. 2008; 11(4): 561-72.

31. Masson LF, McNeill G, Tomany JO, Simpson JA, Peace HS, Wei L, et al. Statistical approaches for assessing the relative validity of a food-frequency questionnaire: use of correlation coefficients and the kappa statistic. Public Health Nutr. 2003; 6(3): 313-21.

32. Willett W. Nutritional epidemiology. $2^{\text {nd }}$ ed. New York: Oxford University Press; 1998.

Recebido em: 30/11/2010 Aprovado em: 26/9/2011 
